\title{
Evaluación y reducción de riesgo en el trabajo de campo*
}

\author{
SUSANN VALLENTIN HJORTH BOISEN**
}

\begin{abstract}
Risk Assessment AND Risk REDUCTION IN FiELDWORK. An analysis of the traditional approach of anthropology on the risks offieldwork and the need to adopt more accurate procedures in high risk zones. This paper explores two approaches that have served in anthropology to develop fieldwork in complex scenarios. Likewise, it explains the risk assessment procedures, the elaboration of mitigation measures and their usefulness for anthropological fieldwork.
\end{abstract}

Key words: methodology, ethnography, acceptance, rapport, prevention

\begin{abstract}
Resumen
Análisis del abordaje tradicional de la antropología en torno a los riesgos del trabajo de campo y la necesidad de adoptar procedimientos más precisos en las zonas de alto riesgo. El artículo examina dos enfoques que han servido en la antropología para desarrollar trabajo de campo en escenarios complejos. Asimismo, explica procedimientos de evaluación de riesgo, la elaboración de medidas de mitigación y su utilidad para el trabajo de campo antropológico.
\end{abstract}

Palabras clave: metodología, etnografía, aceptación, rapport, prevención

\section{Introducción}

$\mathrm{E}$ n meses recientes he realizado trabajo de campo en Tapachula, Chiapas, en la frontera sur de México, investigando el creciente número de centroamericanos que entran al país en busca de refugio. El gran incremento de las solicitudes de asilo en México desde el año 2014 se debe, sobre todo, a la violencia perpetrada por las pandillas contra la población en el Triángulo del Norte de Centroamérica (El Salvador, Guatemala y Honduras). Uno de mis objetivos fue reflexionar en torno a las estrategias de los refugiados para afrontar la inseguridad en su comunidad de origen y los acontecimientos que terminan por desencadenar el desplazamiento de la población. En mi opinión, estos sucesos constituyen puntos de inflexión en la trayectoria personal de los refugiados, que a su vez marcan el agotamiento de los recursos individuales y sociales para hacerle frente a la violencia en sus comunidades.

El tema de las medidas que asume la población para sobrevivir y enfrentar la violencia que la rodea me remitió a otro trabajo de campo que efectué en Ciudad Juárez, Chihuahua, hace pocos años, con los migrantes nahuas del sur de Veracruz que viajan para laborar en las maquiladoras de la frontera norte de México. Ciudad Juárez presenta altos índices de delincuencia, pandillas y crimen organizado. A lo largo de una década, en varias ocasiones pasé meses enteros viviendo en casas de las familias migrantes en esa ciudad, ubicadas en las colonias

\footnotetext{
* Artículo recibido el 17/10/17 y aceptado el 02/01/18.

** Centro de Investigaciones y Estudios Superiores en Antropología Social-Ciudad de México. Juárez núm. 87, col. Tlalpan, Tlalpan, 14000, Ciudad de México <svhb@ciesas.edu.mx>.
} 
marginales -algunas de ellas con elevados niveles de inseguridad-que rodean los parques industriales de la urbe, y recorría frecuentemente con ellos los 2500 kilómetros que separan el sur de Veracruz de la frontera norte de México. A pesar de las evidentes diferencias, reconocí elementos comunes en los relatos de los refugiados centroamericanos y los migrantes nahuas en Ciudad Juárez: el temor a salir a la calle; los toques de queda impuestos por la delincuencia; la autorrestricción para moverse fuera de la casa; la necesidad de evitar la oscuridad de la tarde, de seleccionar las rutas más seguras y de mantener un bajo perfil; así como la sensación de vulnerabilidad, impotencia y, a veces, resignación.

En particular el tema de los refugiados me hizo meditar sobre los límites de las estrategias de afrontamiento de la población en tales contextos de inseguridad, y esto me condujo al trabajo de campo que llevamos a cabo los antropólogos en entornos inseguros y las estrategias que desarrollamos consciente e inconscientemente durante estos trabajos. Me hizo pensar en los posibles límites de estas estrategias y las medidas complementarias que podrían ser útiles para prever y enfrentar estas situaciones de una manera más sistemática. Tal reflexión es el objetivo del presente texto.

Este artículo fue hecho con base en tres fuentes diferentes: mi propia experiencia de campo en múltiples localidades en México, una revisión de la literatura antropológica que discute el tema de los riesgos en campo, la producción respecto del tema de seguridad de algunos autores y organizaciones reconocidos, de donde seleccioné los enfoques que me parecieron más adecuados y útiles para las particularidades y demandas del trabajo de campo antropológico.

La introducción al libro de Raymond Lee, Dangerous Fieldwork, menciona los riesgos usuales que enfrentan los antropólogos en el trabajo de campo:

Un buen número de estudios de campo tanto en sociología como en antropología colocan incluso a los trabajadores de campo más tímidos y retraídos en escenarios y situaciones que son potencialmente peligrosos para su salud y seguridad. Es probable que cualquier lista de peligros potenciales sea larga, pero una lista breve seguramente incluiría los peligros de asalto, violación y robo, el riesgo de infección, accidente y enfermedad, así como las posibilidades de arresto, acoso, abuso verbal y enfrentamiento violento [Lee, 1995: vii].
Muchos de los riesgos que anota Lee han sido una constante por décadas en el trabajo antropológico (véase Howell, 1990). Sin embargo, hay motivos para pensar que este trabajo se ha tornado más peligroso en diferentes partes del mundo después del fin de la guerra fría. Lee (1995: 3) y Sluka (1995: 276) señalan que se han multiplicado los conflictos armados y se han desdibujado los actores tradicionales en ellos. ${ }^{1}$ No obstante, en América Latina, durante la última década, el incremento de la violencia asociada con la delincuencia organizada y las pandillas representa quizá el principal factor de la creciente inseguridad que padecen los investigadores en el trabajo de campo.

Es común que antropólogos experimentados muestren una gran capacidad para resolver situaciones adversas; asimismo suelen compartir exóticas historias ${ }^{2}$ sobre haber salido ilesos de episodios accidentados e imprevistos. Esta cultura profesional considera muchos de los riesgos vinculados con el trabajo de campo como una parte natural y aceptable del proceso de investigación. Incluso, percances menores en campo suelen estimarse un necesario rito de paso para antropólogos novatos (Lee, 1995: 64; Gill, 2004). Creo que, por un lado, podría argumentarse que esta peculiar construcción social del riesgo en la antropología predispone a algunos estudiantes e investigadores a menospreciar los peligros, y que, por otro, esta actitud tiene su fundamento en el reconocimiento de que sin la voluntad de exponerse a los riesgos inherentes a cualquier trabajo de campo éste sencillamente no se podría realizar. Autores como Sluka (1990: 114; 1995: 276), Gill (2004: 3) y Ice, Dufour y Stevens (2015: 129) parecen también advertir este dilema.

El problema aparece cuando la situación que encuentra el investigador en campo lo coloca en un peligro inminente, o bien, la manera de enfrentar los riesgos asociados con un tema o un lugar particularmente peligroso se deja a la improvisación. Por este motivo, los autores especializados concuerdan en que el tema de la seguridad debe formar parte de la fase de preparación y planeación del trabajo de campo, sobre todo en zonas de alto riesgo (Goldstein, 2014: 6; Westmarland, 2002: 39; Jamieson, 2002: 61; Ice, Dufour y Stevens, 2015: 143; Martin-Ortega y Herman, 2009: 227; IFRC, 2009: 12). Sluka, quien efectuó varios trabajos de investigación con movimientos clandestinos en Belfast, durante el conflicto armado en Irlanda del Norte, puntualiza que cuando los

\footnotetext{
1 Las organizaciones humanitarias también han experimentado un incremento de los incidentes de seguridad por estas causas (Van Brabant, 2001: 16; Collinson et al., 2013: 1).

2 Lee (1995: 64) y Ice, Dufour y Stevens (2015: 128) apuntan que, en la academia, este tipo de relatos suelen ser utilizados como cuentos de aventura y gloria y no como una advertencia.
} 
antropólogos se internan en un escenario peligroso "en un grado sustancial, los peligros que enfrentan [...] en su trabajo de campo pueden ser mediados a través de la previsión, la planificación y la maniobra hábil" (1995: 277).

\section{Enfoques de seguridad desde la tradición antropológica}

La antropología ha dado a conocer pocos intentos por sistematizar el tema de riesgos en el trabajo de campo, ${ }^{3}$ aunque varios investigadores reconocidos han desarrollado estrategias personales a partir de sus propias experiencias acumuladas a lo largo de múltiples trabajos en contextos de alto riesgo. Esto posiblemente sea algo común. En lo personal, durante mis estadías de trabajo de campo he implementado con éxito varias estrategias para cuidar la seguridad en entornos inseguros. Éstas contribuyeron a insertarme sin contratiempos durante largos periodos en algunas ciudades de las fronteras norte y sur de México, en los espacios de tránsito de la migración y en otras zonas del país que presentan diversos riesgos.

Después de analizar y comparar mis experiencias con la literatura antropológica en torno al tema, propongo agrupar tales experiencias en dos estrategias principales que he comprobado son útiles para reducir
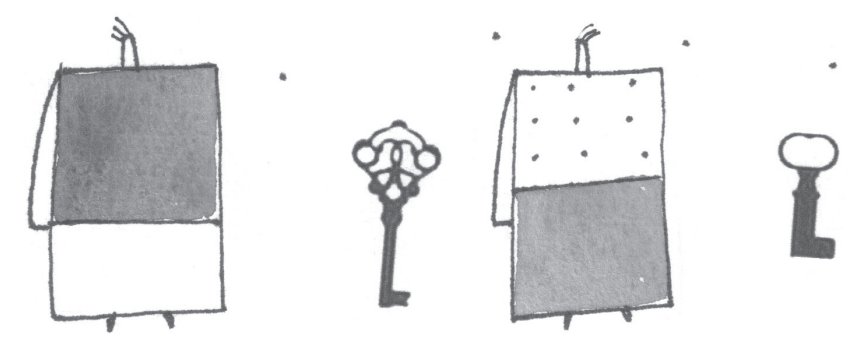

-

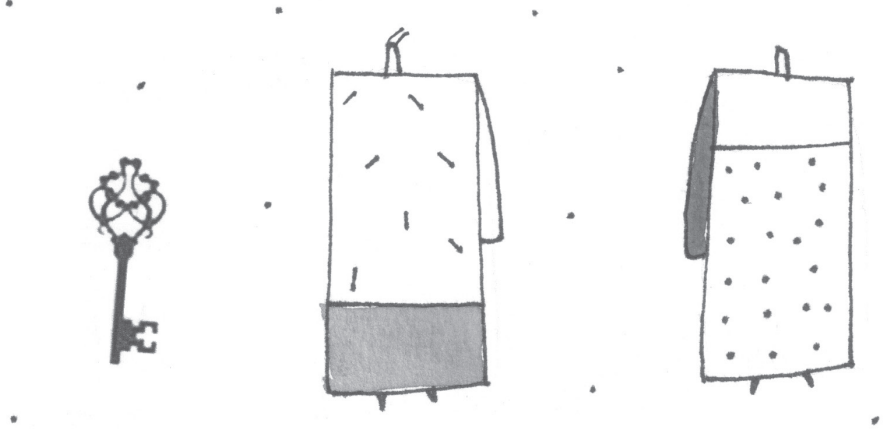

los riesgos en el trabajo de campo. Se trata, por un lado, de una serie de procedimientos antropológicos que aglutino bajo el término estrategia de la aceptación. Por otro lado, existen procedimientos que reúno bajo el concepto enfoque etnográfico, acuñado por Goldstein (2014: 14). Son dos principios que, en la práctica, ya forman parte del trabajo de campo y que, cuando son aplicados correctamente, pueden fortalecer de manera importante la seguridad del investigador.

\section{La estrategia de la aceptación}

Existen varias estrategias comúnmente aceptadas para reducir los riesgos en un escenario, entre las cuales se encuentran las de la aceptación, la protección y la disuasión. Las dos últimas pueden, en general, considerarse impracticables y contraproducentes ${ }^{4}$ en el trabajo de campo antropológico. Sin embargo, el enfoque o estrategia de la aceptación ha sido desarrollado con amplitud desde la literatura especializada como uno de los ejes de seguridad más viables para personal en campo (Van Brabant et al., 2010: 55; Van Brabant, 2000: 60; IFRC, 2009: 16, 20). A diferencia de otras estrategias, esta perspectiva intenta disminuir o eliminar las amenazas aumentando la aceptación en un contexto particular (Van Brabant et al., 2010: 55; véase también IFRC, 2009: 21).

En muchos sentidos, el enfoque de la aceptación no es nuevo para la antropología, que ha abordado el tema desde un punto de vista metodológico durante décadas. Tradicionalmente, para la antropología, la aceptación de la comunidad hacia el investigador es vital para la ejecución exitosa del trabajo de campo, y para la generación de datos. En la antropología, el equivalente a la estrategia de la aceptación es el desarrollo del rapport en el escenario, entendido éste como el establecimiento de la confianza suficiente con los informantes (Taylor y Bogdan, 1996: 55). El desarrollo del rapport representa un fundamento indispensable para el trabajo etnográfico y las técnicas que lo acompañan: la observación participante y la realización de entrevistas en sus diferentes modalidades (Goldstein, 2014: 3). En mi opinión, en la antropología, una estrategia de seguridad basada en la aceptación debe, idealmente, aparecer como otro subproducto del rapport desarrollado en el trabajo de campo. Creo que quizá éste sea uno de los principales recursos para la seguridad del etnógrafo en escenarios complejos.

3 Una excepción es el trabajo de Nancy Howell (1990).

4 En Paradoxes of Presence, Collinson et al. (2013: 12) discuten las implicaciones negativas del creciente énfasis sobre las medidas de protección y disuasión de las organizaciones humanitarias internacionales, en detrimento de la aceptación. 
Trabajar el rapport en escenarios inseguros, sin embargo, no está exento de complicaciones. Goldstein (2014) menciona con gran acierto que el desarrollo del rapport es en especial difícil en contextos de alta violencia. Por este motivo, se ha señalado que es indispensable ser en extremo vigilantes del proceso de identificación ${ }^{5}$ o creación del rol de campo en tales entornos (Brown, 2009: 214; Sluka, 1995: 283; Lee, 1995: 75). En este sentido, Sluka (1995: 284) subraya la importancia de evitar a toda costa la asignación de un rol que puede ser visto como una amenaza para las personas en el escenario y buscar activamente encajar en roles más seguros y aceptados en la comunidad. Es menester trabajar de un modo ininterrumpido para establecer y reafirmar de manera permanente la credibilidad del investigador y disipar con eficacia las dudas sobre sus intenciones. Para evitar sospechas, todos los autores concuerdan en que tal vez la mejor estrategia es ser lo más abierto posible en torno a las intenciones y objetivos de la investigación con las personas y actores en el escenario (Goldstein, 2014: 8; Sluka, 1990: 118 y 1995: 284-285).

Lo que yo he vivido se une al consenso de estos autores. En la sierra de Soteapan, al mismo tiempo que trabajé el rapport con la población, logré progresivamente construir cierta confianza con los intermediarios que llevaban los migrantes. El tiempo se encargó de mostrar que mi presencia no dificultaba su trabajo y eso ayudó mucho en la construcción de rapport. Esto implicó una importante ventaja para estudiar el tema de la migración, viajar por las rutas de tránsito entre el sur de Veracruz y la frontera norte, así como para mi movilidad dentro de las colonias periféricas de Ciudad Juárez. Debido a las características de la investigación, el desarrollo exitoso del rapport y del proceso de identificación fueron claves para la amplitud y profundidad del trabajo etnográfico.

En mi experiencia, la actitud y capacidad individual del investigador para construir rapport se convierte en elemento fundamental para su seguridad en campo. Sluka (1995: 287) hace una observación muy parecida en sus comentarios sobre su trabajo en Belfast, donde precisa que identificarse con los intereses, entender los problemas y preocupaciones de la gente, y actuar en concordancia con ello, puede ser muy efectivo para reducir el peligro en el trabajo de campo. Por su parte, Hilhorst et al. (2016: 9) puntualizan:

tratar a las personas con respeto ayuda a construir la relación de confianza que se necesita para obtener información confiable y persuadir a las personas para que compartan sus historias. Fomentar relaciones respetuosas afecta directamente la seguridad de los investigadores, ya que mejora el acceso a información referente a la seguridad y puede significar que las personas son más propensas a advertirle o ayudarle en caso de peligro.

Considero que el enfoque de la aceptación es acaso la mejor estrategia de reducción de riesgo de que dispone el investigador de campo. Sin embargo, una consecuencia de esta intensa inmersión que requiere el trabajo etnográfico es que, en mi opinión, hace muy difícil aplicar cualquier estrategia alternativa de seguridad. Si el rapport y la estrategia de la aceptación representan la unión perfecta entre las técnicas cualitativas de investigación de campo y las técnicas de mitigación de riesgos, también lleva implícita sus propios límites. La estrategia de la aceptación acerca al antropólogo a la población, quien, en el mejor de los casos, lo protege, pero al hacerlo inhabilita el uso de estrategias alternativas y ahí radica posiblemente su mayor debilidad.

\section{El enfoque etnográfico}

Durante el trabajo de campo y mediante la convivencia cotidiana, los investigadores pasan por un progresivo proceso de aprendizaje de las normas de conducta del grupo. Años de entrenamiento en la observación participante hacen del antropólogo un buen alumno de las costumbres locales, lo cual es otra gran ventaja para enfrentar los riesgos que derivan de causas ambientales y altos niveles de inseguridad.

En este sentido, conviene estudiar y aprender los mecanismos que usa la gente en el escenario para aminorar los riesgos del día a día. Esto puede enseñar al investigador mucho sobre cómo sobrevivir en el escenario. En lo personal, he utilizado esta estrategia para abordar los retos cotidianos de movilidad en diferentes sitios de trabajo en Tapachula, en la sierra del sur de Veracruz, en las calles de Ciudad Juárez y en muchos otros contextos. Durante mi trabajo en campo he observado los ritmos, rutas y horarios del movimiento de la gente, sus formas de evitar exponerse en ciertas zonas y a los asaltos, el modo de mirar, de pararse, de hablar, y los momentos para guardar silencio, ser asertivo o dejar de serlo, y me he encontrado una y otra vez adoptando estas conductas al realizar mi labor. No me cabe duda de que adoptar

5 El término identificación se usa aquí para caracterizar el proceso mediante el cual las personas en el escenario tienden a encasillar al investigador dentro de determinados roles disponibles en el contexto local. 
algunas de estas costumbres mitiga los riesgos, porque el antropólogo aprende de la experiencia colectiva sobre la mejor manera de evitar problemas en el escenario.

Algunos autores señalan este método como una estrategia fundamental para hacerle frente a un entorno inseguro. Goldstein (2014: 5, 14) llama enfoque etnográfico a dicho acercamiento. Éste también puede entenderse como una estrategia de imitación, adopción o emulación, basada en la capacidad del investigador para observar las conductas locales y adoptarlas para reducir los riesgos a los que está expuesto. De modo similar, a partir de su trabajo de campo con los niños de la calle en Brasil, Kovats-Bernat (2002: 214) subraya la necesidad de desarrollar lo que llama localized ethics, o una ética localizada, consistente en seguir el consejo y las recomendaciones de la población local en cuanto a los temas que no son prudentes comentar con otros, además de adoptar los patrones locales de conducta para protegerse a sí mismo y a las personas que le rodean en su trabajo de campo.

Aunque no hay duda sobre la utilidad de este enfoque para mantener a salvo al estudioso, juzgo conveniente apuntar que estos autores no discuten el problema de que el etnógrafo nunca será considerado por los actores locales como un miembro de la comunidad. Por otro lado, es evidente que las actividades del investigador de campo son distintas de las de la población local, y sus rutinas, contactos y recorridos pueden diferir mucho de los de ella, por lo que los riesgos no siempre se podrán encarar de igual modo en que lo hacen las personas que viven en el lugar. Por lo tanto, las medidas locales pueden convertirse en limitaciones, o pueden, en sí mismas, ser insuficientes cuando el etnógrafo necesita desplegar sus herramientas de investigación.

También hay que destacar que las estrategias de la población para resolver situaciones de inseguridad no siempre resultan muy efectivas. La gente puede basar su conducta en información incompleta y contradictoria, en rumores o en nociones falsas. Raymond Lee (1995: 29) menciona casos en donde la población emplea medidas de protección basadas en la magia, lo cual, según mi propia experiencia etnográfica, es una práctica bastante extendida en muchos entornos con elevados niveles de inseguridad. No obstante, me parece claro que la eficacia de las estrategias locales de afrontamiento tiene un límite. De hecho, los informantes también sufren accidentes, se enferman y son víctimas de delitos. Las entrevistas que he realizado con refugiados que huyen de la violencia pandillera en Centroamérica representan un patente ejemplo de cómo las múltiples estrategias para hacerle frente a la violencia en sus lugares de origen llegan a agotarse y fallar-con un alto costo-en un ambiente de seguridad en constante deterioro. Por estos motivos, puede ser aconsejable reflexionar sobre las medidas locales adoptadas, su ámbito de aplicación y sus límites, antes de imitarlas acríticamente durante el trabajo de campo.

\section{Métodos de análisis de riesgo}

A continuación discutiré algunas aproximaciones de análisis de riesgo y medidas de mitigación, aceptadas por lo general, las cuales pienso pueden adaptarse de manera ventajosa para el trabajo de campo antropológico y así fortalecer las condiciones de seguridad en él, complementando las estrategias antes analizadas.

Todos los enfoques comparten ciertas nociones clave. En primer lugar, se encuentra el concepto de riesgo. Entre varias definiciones existentes opto por la de Van Brabant et al. (2010: 28), quienes lo definen como "la probabilidad de que ocurra algo dañino, y el alcance de ese daño, si lo hace". Por otra parte, es dable entender una amenaza (threath) como cualquier cosa que puede causar daño (Van Brabant et al., 2010: 29; IFRC, 2009: 37) o como la posibilidad de que algo o alguien dañe la integridad física o moral o la propiedad de una persona (Есно, 2004: 11). El concepto de amenaza está vinculado con el de vulnerabilidad, que es la posibilidad de sufrir un daño (Eguren y Caraj, 2009: 29) y que se puede concebir también como el nivel de exposición a una amenaza (IFRC, 2009: 37; ЕСHO, 2004: 11).

Muchos autores concuerdan en que existen dos tipos de amenazas básicas: a) las que emanan del entorno natural y del social, que son eventos de diferente tipo producidos en la zona y que no están directamente dirigidos a la persona, pero que la pueden afectar; y b) aquellas donde la presencia del investigador provoca hostilidad, agresión o violencia (Lee, 1995: 3; Ice, Dufour y Stevens, 2015: 129). Al primer tipo de amenazas se les puede llamar incidentales (Eguren y Caraj, 2009: 29) o ambientales (Lee, 1995: 3), mientras que al segundo tipo situacionales.

Discutiré tres acercamientos, que es factible utilizar de manera independiente o secuenciada, de acuerdo con las características y los riesgos asociados con el proyecto de investigación. El primer procedimiento, el análisis de contexto, da cuenta de la presencia de diversos factores de riesgo en un contexto determinado. El segundo, el análisis situacional, se enfoca casi exclusivamente en los factores situacionales que pueden implicar un riesgo potencial para el investigador. Por último, la evaluación de riesgo es una herramienta 
más compleja que examina los niveles de riesgo y las medidas de mitigación, tanto para las amenazas incidentales como situacionales.

\section{Análisis de contexto}

El análisis de contexto (Van Brabant et al., 2010: 29; IFRC, 2009: 17) es un procedimiento que permite entender los peligros de diferente naturaleza que podrían estar presentes en un lugar. Por lo tanto, supone la recopilación, lo más completa posible, de información del escenario y la zona geográfica donde se va a realizar el trabajo. Van Brabant, entre otros, señala que se trata de buscar los elementos que podrían ser relevantes para producir una comprensión general de la zona donde se va a trabajar y, a partir de ella, identificar las principales amenazas, tanto naturales como humanas (Van Brabant et al., 2010: 39; FEMA, 2016). También es usual que los antropólogos especializados recomienden una comprensión amplia y clara del entorno desde la fase de planeación (Howell, 1990: 94, 185; Lee, 1995: 73; Mertus, 2009: 170; Sluka, 1990: 121).

Se debe investigar y analizar, por una parte, la situación geográfica, la propensión a los desastres
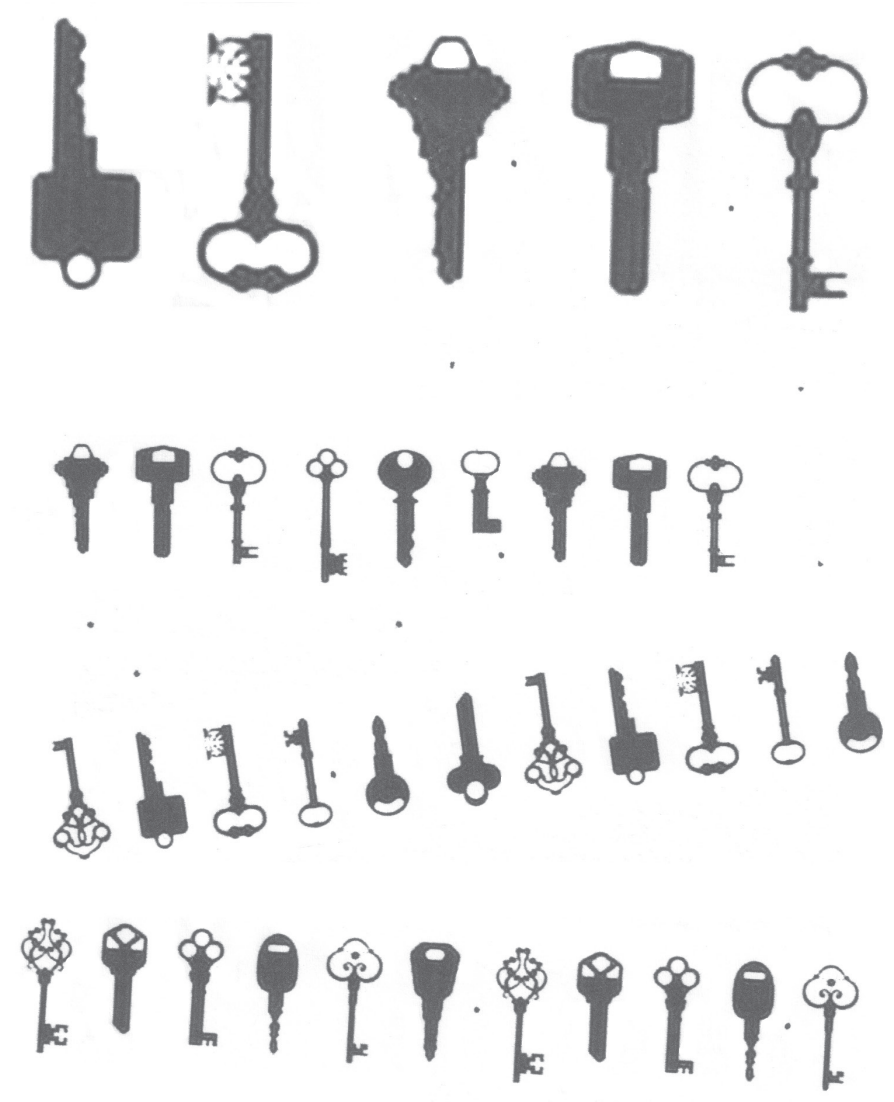

naturales y las características e impacto de éstos en el pasado. Asimismo, hay que indagar sobre la existencia de epidemias, animales venenosos o vectores transmisores de enfermedades; las características de asentamiento humano; la infraestructura; el transporte; las comunicaciones; los servicios y la presencia de hospitales y clínicas. Por otra parte, hay que examinar y comprender los fenómenos sociales y políticos en el escenario. Esto conlleva escrudiñar sobre la historia; la estructura social; los rasgos del grupo étnico o social de estudio; la naturaleza, carácter e historia de eventuales conflictos que están presentes; las políticas regionales; los grupos que disputan el poder y lo privativo de la economía local. Además se debe averiguar sobre la naturaleza de las actividades de la delincuencia, y de los incidentes de violencia en la zona (Hilhorst et al., 2016: 30; Ice, Dufour y Stevens, 2015: 143; Mertus, 2009: 171). Por sus atributos, considero que el análisis de contexto es un procedimiento particularmente útil para establecer las amenazas incidentales, que son las que el investigador comparte con la población en el escenario.

\section{Análisis situacional}

El análisis situacional (Van Brabant, 2000; Mertus, 2009: 170) se centra en el estudio de los principales actores o grupos que potencialmente podrían afectar la seguridad o el trabajo en el escenario. En este sentido, es posible ubicar al análisis situacional como una parte especializada del análisis de contexto, pero creo que es conveniente profundizar en este procedimiento por separado.

Así, el análisis situacional tiene cuatro componentes fundamentales. El primero consiste en identificar y elaborar una lista de los diferentes actores que existen en la zona y de los grupos locales y externos que se presume pueden ejercer una influencia sobre la situación local o el trabajo de campo. El segundo es examinar las características de cada uno de estos actores en torno a sus objetivos, intereses, estrategias y métodos (Van Brabant et al., 2010: 33; Van Brabant, 2000: 58; Hilhorst et al., 2016: 30).

El tercero es analizar, hasta donde sea posible, las relaciones entre estos actores, que algunos definen como la microdinámica local (Eguren y Caraj, 2009: 19). Debe recordarse que, a veces, múltiples conflictos se encuentran entretejidos (Van Brabant et al., 2010: 31). En mi opinión, es esencial evaluar el papel que juega el grupo étnico, la comunidad o el grupo social con quien se trabajará dentro de estas microdinámicas locales. Este papel tendrá consecuencias directas para 
las condiciones del trabajo de campo, pues el investigador estará expuesto a situaciones que derivan de su presencia y posición dentro de estas microdinámicas. Por último, hay que intentar comprender la forma en que los actores y la situación local pueden afectar al investigador y al trabajo que se propone realizar. En este momento del análisis es fundamental evaluar si la presencia del investigador o su trabajo serían vistos de manera negativa por los actores locales y si este hecho supone un riesgo.

\section{Evaluación de riesgo}

Una vez efectuados el análisis de contexto y el situacional, si se estima conveniente debido al nivel de amenazas, puede llevarse a cabo un procedimiento de evaluación de riesgo (risk assessment). Éste examina sistemáticamente las amenazas presentes en el lugar, las vulnerabilidades de la gente hacia ellas, los posibles impactos negativos de la materialización de cada una de las amenazas y las probabilidades de que ocurran. Por último, a partir de lo anterior, establece las medidas correspondientes de mitigación o reducción de riesgo (IFRC, 2009: 37; Norman, 2016: 10).

Además de los conceptos de amenaza, vulnerabilidad y riesgo ya discutidos, la evaluación de riesgo incluye también la noción de impacto, o consecuencia, definida como el daño potencial que puede causar la materialización de una amenaza determinada. Como se dijo antes, es dable describir el riesgo como la probabilidad de que ocurra un evento dañino y la extensión del posible daño causado por él. También puede entenderse como la combinación de una amenaza y la vulnerabilidad que existe hacia esa amenaza (Van Brabant et al., 2010: 46). Para Van Brabant et al. (2010: 28), las medidas de mitigación de riesgo son las acciones emprendidas para disminuir la amenaza o la vulnerabilidad hacia ella. A su vez, la vulnerabilidad se aminora reduciendo la exposición al evento o su impacto, o ambos.
La evaluación de riesgo consiste básicamente en cinco pasos fundamentales: 1) consideración de las amenazas, 2) consideración del impacto, 3) estimación de la probabilidad de ocurrencia, 4) análisis del riesgo y 5) diseño de las medidas de mitigación. La forma más sencilla de realizar este procedimiento es mediante la elaboración de un cuadro (cuadro 1 ).

La primera columna debe ser una lista de las amenazas de diferentes fuentes, tanto incidentales como situacionales, capaces de afectar al investigador y al trabajo de investigación en el escenario. Es importante enlistar todas las amenazas naturales y humanas susceptibles de ocurrir en el lugar.

Una vez terminada la primera columna, en la segunda debe anotarse la localización de cada amenaza (el lugar donde puede ocurrir). En la tercera se debe estimar la gravedad, o la consecuencia, del impacto, en caso de que se materialicen estas amenazas. Se trata de identificar la peor consecuencia de cada una. El impacto puede presentarse en forma de un daño físico, emocional, financiero, daño o pérdida de equipo o efectos negativos para la realización del proyecto de investigación. El impacto del evento se puede calificar dentro de una escala con las opciones bajo, medio, alto y muy alto.

Después de evaluar el impacto potencial de todos los eventos, en la cuarta columna debe estimarse la probabilidad de que ocurra cada uno de ellos. La probabilidad es, en buena medida, una cuestión de juicio personal. Para el caso de la antropología, es difícil obtener indicadores precisos en muchas de las zonas donde típicamente se realiza trabajo de campo. En estos casos, y con base en la información recabada y la experiencia de campo, se puede calificar la probabilidad de ocurrencia del evento en una escala de baja, media, alta o muy alta.

En la quinta columna debe estimarse el nivel de riesgo, el cual es una expresión de la vulnerabilidad hacia una amenaza determinada. Para evaluarlo, puede consultarse la matriz en el cuadro 2 , donde se cruzan, por un lado, el nivel del impacto y, por el otro, la

\section{Cuadro 1. Evaluación de riesgo y determinación de medidas de mitigación}

\begin{tabular}{|l|l|c|c|c|c|c|}
\hline $\begin{array}{c}\text { Evento } \\
\text { (amenaza) }\end{array}$ & Localización & $\begin{array}{c}\text { Impacto } \\
\text { (bajo, medio, } \\
\text { alto, muy alto) }\end{array}$ & $\begin{array}{c}\text { Probabilidad } \\
\text { (baja, media, } \\
\text { alta, muy alta) }\end{array}$ & $\begin{array}{c}\text { Nivel de riesgo } \\
\text { (bajo, medio, alto, } \\
\text { muy alto) }\end{array}$ & $\begin{array}{c}\text { Medidas de } \\
\text { mitigación }\end{array}$ & $\begin{array}{c}\text { Riesgo residual } \\
\text { (bajo, medio, alto, } \\
\text { muy alto) }\end{array}$ \\
\hline & & & & & & \\
\hline & & & & & & \\
\hline
\end{tabular}

Fuente: Elaboración propia con base en Van Brabant et al. (2010: 51). 
probabilidad de ocurrencia del evento. La combinación de estos valores establece el nivel de riesgo.

Ya establecido el nivel de riesgo, el siguiente paso es seleccionar las medidas para su reducción o mitigación, las cuales, como se ha aclarado, representan el conjunto de operaciones de reducción de la exposición (disminuye la probabilidad de que ocurra el evento y son principalmente medidas de prevención) y del impacto (frente a una amenaza que se ha materializado). Estas últimas también son conocidas como medidas de contingencia (Van Brabant et al., 2010: 50). Las medidas de mitigación se asentarán en la sexta columna del cuadro 1 .

Por poner un ejemplo común de la antropología, vacunarse y realizarse un chequeo médico son medidas preventivas que reducen la exposición y la probabilidad de tener una enfermedad grave durante el trabajo de campo, mientras que llevar un botiquín de viaje, tener los datos del seguro médico a la mano y conocer la ubicación de los centros de salud representan medidas de reducción de impacto. En conjunto constituyen las medidas de mitigación de riesgo en el tema de salud.

Al seleccionar medidas adecuadas de mitigación se reduce automáticamente la vulnerabilidad frente a la amenaza y, por lo tanto, el riesgo (ЕСHO, 2004: 11). Por ello, una vez que se han escogido las medidas de mitigación, es una buena práctica volver a estimar el nivel de riesgo. Esto se conoce como riesgo residual (Van Brabant et al., 2010: 50), el cual se anotará en la última columna del cuadro 1 .

Merece recalcarse que, en caso de trabajar en escenarios de alto riesgo, la selección de las medidas de mitigación debe formar parte de un plan individual de seguridad, como han recomendado algunos autores (Martin-Ortega y Herman, 2009: 227; Mertus, 2009: 173), el cual debe contener las medidas de reducción de exposición y de impacto, así como un plan de evacuación. Este plan individual se debe prever desde la fase del diseño de la investigación.

\section{Los componentes individuales del riesgo y su evolución}

Varios autores concuerdan en que las vulnerabilidades tienen un componente individual (Van Brabant et al., 2010: 52; Jamieson, 2002: 63). Otros hablan de factores como género, edad, identidad, nacionalidad, filiación religiosa, orientación sexual y otros más pueden colocar la vulnerabilidad individual por arriba o debajo de la media en un escenario determinado (Ice, Dufour y Stevens, 2015: 144; Martin-Ortega y Herman, 2009: 236). Cabe apuntar, como lo sabe la antropología desde hace mucho, que estos factores siempre influyen sobre el trabajo de campo y el modo en que los informantes reaccionan ante el investigador (Hammersley y Atkinson, 1994: 100). Existe una amplia experiencia desde la academia en abordar estas características en relación con sus implicaciones metodológicas, el acceso, el proceso de identificación y la construcción del rol del investigador en campo (Taylor y Bogdan, 1996: 53; Hammersley y Atkinson, 1994: 117), pero, con excepción del género, todavía es poco lo que se ha reflexionado en torno al tema desde el punto de vista de los riesgos. En general, creo que la forma correcta de abordar el tema del riesgo y los factores individuales de riesgo es analizar las percepciones que pueden existir tanto en el grupo como en el escenario, luego determinar los diferentes riesgos derivados de éstas y seleccionar las medidas correspondientes de manejo y mitigación de acuerdo con el contexto.

Por otra parte, ciertas vulnerabilidades pueden ser contrastadas por las capacidades (United Nations, 2013: 116), las cuales son individuales y pueden entenderse como "los puntos fuertes y los recursos a los que puede acceder" una persona (Eguren y Caraj, 2009: 31). En mi opinión, en la antropología existen algunos factores que pueden fortalecer estas capacidades individuales. Juega, sin duda, un papel cardinal la experiencia previa del investigador en el trabajo de campo. También son relevantes aspectos como el

Cuadro 2. Matriz de evaluación de riesgo

\begin{tabular}{|c|l|c|c|c|c|}
\hline \multicolumn{2}{|c|}{} & \multicolumn{4}{c|}{ Impacto } \\
\cline { 3 - 6 } \multicolumn{2}{c|}{} & Bajo & Medio & Alto & Muy alto \\
\hline \multirow{3}{*}{ Probabilidad } & Muy alta & Medio & Alto & Muy Alto & Muy alto \\
\cline { 2 - 6 } & Alta & Medio & Alto & Alto & Muy alto \\
\cline { 2 - 6 } & Media & Bajo & Medio & Alto & Alto \\
\cline { 2 - 6 } & Baja & Bajo & Bajo & Medio & Medio \\
\hline
\end{tabular}

Fuente: Elaboración propia con base en Van Brabant et al. (2010: 49). 
conocimiento del grupo y de la cultura local, las redes de relaciones interpersonales desarrolladas con anterioridad en el lugar y el conocimiento de la zona. Estos factores pueden representar importantes recursos personales de mitigación de riesgo en el momento de decidir desarrollar un trabajo de campo en escenarios inseguros. Podría asimismo ser una ventaja la participación en equipos de investigadores con presencia en la misma zona de manera continua.

Otro elemento por considerar es que los riesgos suelen ser más altos al comienzo del trabajo de campo, sobre todo en entornos nuevos, cuando el investigador tiene un conocimiento limitado del lugar y cuenta con pocos contactos locales. Trazando un paralelo con las condiciones de trabajo de las organizaciones humanitarias, es dable afirmar que el periodo inicial de llegada a campo representa el momento de mayor vulnerabilidad (Ice, Dufour y Stevens, 2015: 135; IFRC, 2009; Lee, 1995: 74). Una vez que se ha desplegado un conocimiento más completo de las dinámicas sociales del escenario, se ha establecido el rapport con la población y se dispone de una red de relaciones interpersonales en la comunidad, en condiciones normales, el nivel de riesgo se reduce.

Vale la pena tener en cuenta que, en contextos de elevados niveles de inseguridad, las microdinámicas locales pueden transformarse con rapidez. Por ello, el riesgo adquiere un carácter cambiante y fluido (fluid) (Ice, Dufour y Stevens, 2015: 135; Eguren y Caraj, 2009: 27). Así, durante el trabajo de campo también van modificándose, de manera paralela, las vulnerabilidades del investigador. Estos cambios a veces son evidentes y a veces pasan casi inadvertidos. Por este motivo es recomendable mantener actualizada la información sobre la situación local durante el trabajo de campo y analizar con oportunidad las transformaciones producidas. También es de suma trascendencia subrayar que, en entornos de altos niveles de inseguridad, el investigador debe desarrollar y cultivar una gran flexibilidad y amplia disposición para adaptarse a los cambios generados en el entorno. ${ }^{6}$

Propongo incorporar en el análisis de seguridad el concepto de punto de inflexión, el cual es común en el análisis de trayectorias. Utilicé este concepto en el análisis de las trayectorias de refugiados con el propósito de caracterizar el incidente final agudo (que normalmente representa el punto culminante de una serie de incidentes anteriores) que los obliga a desplazarse de su lugar de origen y que coincide con el agotamiento de las medidas de afrontamiento. Sugiero emplear el concepto de punto de inflexión en el análisis de seguridad para describir el momento en que los cambios generados en el entorno o una acumulación de incidentes previos producen un incidente de seguridad agudo, esto es, cuando las medidas de mitigación de riesgo asumidas resultan insuficientes y se encuentran rebasadas. Esto entraña un peligro inminente, y la retirada del lugar debe ocurrir antes de llegar a este punto.

Los cambios que condujeron al punto de inflexión pueden implicar que la persona se ha convertido en un objetivo para los actores locales (amenaza situacional) o que los cambios acumulativos en la situación local han originado un incremento general de los riesgos en el escenario (amenazas incidentales o ambientales), o ambos. La meta en campo debe ser detectar de manera oportuna estos cambios y evitar llegar a este punto de inflexión. En caso de existir una amenaza situacional, debe señalarse que ninguna de las estrategias discutidas en el presente artículo es suficiente y se recomienda alejarse del escenario.

\section{¿Cuáles son los riesgos aceptables?}

Antes de decidir trabajar en un escenario con riesgos elevados, hay que definir cuál es el nivel de riesgo aceptable para las personas participantes en la investigación. En el caso de la antropología, deben considerarse tanto la integridad física y emocional del investigador, de los informantes y de otros participantes, como la posibilidad de llevar a cabo un trabajo de investigación que cumpla con la rigurosidad necesaria. Irse a campo sólo para eventualmente darse cuenta de que hay que relocalizar el trabajo puede ser una experiencia costosa y causar un grave retraso en el proyecto.

Hay varios factores que tener en cuenta al momento de decidir si un determinado nivel de riesgo es aceptable o no. Por un lado, las instituciones pueden tener lineamientos y políticas propios en torno a los riesgos aceptables para estudiantes e investigadores. Por otro lado, en una tradición antropológica donde existen pocos lineamientos institucionales alrededor de este tema, el componente personal es muy importante.

Para Sluka, reconocido autor que trabaja el tema, la cuestión del riesgo aceptable pasa sobre todo por una reflexión personal:

Decida si está preparado para aceptar los riesgos involucrados [...] Reflexione acerca de lo que sería un nivel aceptable de peligro. Supongo que la mayoría de los investigadores no están dispuestos a dar sus vidas por su

${ }^{6}$ Para este tema véanse también Kovats-Bernat (2011: 210) y Sluka (1990: 122). 
investigación y se retiran a un terreno más seguro si surge una amenaza directa a la vida o la integridad física [Sluka, 1995: 282].

Martin-Ortega y Herman (2009: 236) coinciden con Sluka cuando afirman que "cada investigador debe decidir por sí mismo hasta dónde está dispuesto a llegar para protegerse y el umbral de riesgo inaceptable", mientras que Howell (1990: 186) indica que la selección del lugar de campo debe estar de acuerdo con las habilidades y preferencias personales del investigador para enfrentar los riesgos. Para todos estos autores, la percepción de lo que es un riesgo aceptable o no es una consideración muy individual, como también lo son la tolerancia o la aversión a los riesgos (Ice, Dufour y Stevens, 2015: 149). Sin embargo, Kovats-Bernat (2002: 210) asume una posición más analítica. Según este autor, lo primordial es realizar una ponderación objetiva entre riesgos existentes versus el valor de los datos que se desean obtener. Para Kovats-Bernat se trata, en otras palabras, de que los resultados esperados de la investigación estén equilibrados con el nivel de exposición a los riesgos.

Desde mi punto de vista, no es conveniente llevar a cabo un trabajo de campo para el cual no se han sopesado y aceptado previamente los riesgos. No sólo puede ser contraproducente encontrarse con peligros que no se anticiparon, también puede tener consecuencias negativas para la investigación. Es probable que una persona que no se encuentra cómoda con determinado nivel de riesgo llegue a limitar las actividades de investigación en el escenario para no exponerse a los peligros, reales o percibidos, o que el estrés emocional restrinja la efectividad en el trabajo etnográfico $^{7}$ o incremente su vulnerabilidad. Esta consideración incluye los riesgos emocionales, que en la actualidad son más reconocidos en la antropología (véanse Castro Neira, 2017: 65; Lankshear, 2002: 81; Lee-Treweek y Linkogle, 2002: 13; Martin-Ortega y Herman, 2009: 237; Mertus, 2009: 166; Nordstrom y Robben, 1995: 13).

Por último, cabe recordar que un trabajo de campo académico requiere la aplicación de una serie de procedimientos rigurosos para la construcción de datos, sin los cuales carece de validez científica. En el caso de la investigación antropológica, el despliegue de las herramientas de investigación incluye actividades como múltiples desplazamientos en la zona, observación participante, búsqueda de informantes y efectuar entrevistas. Por lo tanto, dentro de la ponderación de los riesgos, deben evaluarse de manera destacada las posibilidades de realizar un trabajo de campo de una forma rigurosa y científicamente válida en el escenario elegido.

\section{Conclusiones}

En la actualidad, la investigación de campo se realiza en entornos con una creciente inseguridad e inestabilidad, los cuales generan problemas prácticos para el despliegue de las actividades de investigación. La mayoría de los investigadores desarrollamos nuestros propios métodos para hacer frente a situaciones de inseguridad; éstos suelen ser empíricos y están basados en un proceso acumulativo de experiencias personales en diferentes contextos. Algunos antropólogos especializados han publicado sus vivencias de modo individual, pero todavía existen pocos esfuerzos de sistematización en el tema. Para avanzar en este análisis, propongo agrupar los métodos que usamos habitualmente los antropólogos en dos estrategias básicas: la estrategia de la aceptación y el enfoque etnográfico, ambos discutidos en el presente texto.

Si bien estas dos estrategias mejoran la seguridad en el trabajo de campo, en ciertos contextos pueden resultar insuficientes. Por tal razón, propongo incluir en la etapa de planeación algunas herramientas de análisis y evaluación de riesgo, que mejoran la capacidad de prevención y mitigación de riesgo en el trabajo de campo y proporcionan elementos para ponderar con oportunidad los niveles de riesgo aceptables en los proyectos. La incorporación de estos procedimientos analíticos y de reducción de riesgo en la fase de planeación y durante el trabajo de campo contribuye a mejorar las condiciones de seguridad del estudioso $\mathrm{y}$, por lo tanto, aquellas para la ejecución exitosa del proyecto de investigación.

\section{Fuentes}
BRABANT, KOENRAAD VAN
2000 Operational Security Management in Violent Environments, Humanitarian Practice Net- work-Overseas Development Institute (Good Practice Review, 8), Londres, 354 pp.
BRABANT, KOENRAAD VAN
2001 Mainstreaming the Organisational Management of Safety and Security. A Review of Aid Agency

\footnotetext{
7 Lee (1995: 14) establece que el temor puede hacer que el investigador omita, olvide o distorsione información, o se distancie emocional y físicamente de las situaciones de campo.
} 
Practices and a Guide for Management, Overseas Development Institute (Humanitarian Policy Group Report, 9), Londres, 78 pp.

Brabant, KoEnRAaD VAN ET AL.

2010 Operational Security Management in Violent Environments, edición revisada, Humanitarian Practice Network-Overseas Development Institute (Good Practice Review, 8), Londres, $301 \mathrm{pp}$.

Brown, Stephen

2009 "Dilemmas of Self-representation and Conduct in the Field", en Chandra Lekha Sriram, John C. King, Julie A. Mertus, Olga Martin-Ortega y Johanna Herman (eds.), Surviving Field Research. Working in Violent and Difficult Situations, Routledge, Nueva York, pp. 213-226.

CAstro Neira, Yerko

2017 "Etnografía de la violencia. Dilemas para hacer y pensar las etnografías en zonas de guerra, violencia y conflicto", en Yerko Castro Neira y Adèle Blazquez (coords.), Micropolíticas de la violencia. Reflexiones sobre el trabajo de campo en contextos de guerra, conflicto y violencia, Laboratorio Mixto Internacional (Cuadernos de Trabajo de meso, 5/2017), México, pp. 6070 <https://f.hypotheses.org/wp-content/ blogs.dir / 1966 / files / 2017/09/CUADERNOMESO5-FINAL_040917-ok.pdf> [12 de septiembre de 2017].

Collinson, Sarah, Mark Duffield, Carol Berger,

Diana Felix da Costa y Karl Sandstrom

2013 Paradoxes of Presence. Risk Management and Aid Culture in Challenging Environments, Humanitarian Policy Group-Overseas Development Institute, Londres, 38 pp. <https: / / www.odi. org/publications / 7514-paradoxes-presencerisk-management-and-aid-culture-challengingenvironments> [ 10 de septiembre de 2017].

ECHO

2004 Generic Security Guide for Humanitarian Organisations, European Commission's Directorate-General for Humanitarian Aid, Bruselas, 152 pp. <https: / / www.eisf.eu/library/ generic-security-guide-for-humanitarianorganisations / $>$ [30 de mayo de 2016].

Eguren, Luis EnRigue y María Caraj

2009 Nuevo manual de protección para defensores de derechos humanos, Oficina Europea de Peace Brigades International / Frontline, Dublín, 214 pp. <http: / / protectioninternational.org/es / publication / nuevo-manual-de-proteccionpara-defensores-de-derechos-humanos / > [2 de junio de 2016]

FEMA

2016 Emergency Planning, U.S. Federal Emergency Gill, Hannah E. Management Agency, Washington, 158 pp.

2004 "Finding a Middle Ground between Extremes. Notes on Researching Transnational Crime and Violence", en Anthropology Matters, vol. 6, núm. 2, pp. 1-9<https: / / anthropologymatters. com/index.php/anth_matters/article/view/ $100 / 196>$ [ 12 de junio de 2017].

Goldstein, Daniel M.

2014 Qualitative Research in Dangerous Places: Becoming an "Ethnographer" of Violence and Personal Safety, Drugs, Security and Democracy Program, Working Papers on Research Security, núm. 1, Social Science Research Council <https: / /www.ssrc.org/pages / qualitativeresearch-in-dangerous-places-becoming-anethnographer-of-violence-and-personal-safety> [2 de junio de 2017].

Hammersley, Martyn

y PAUl AtKinson

1994 Etnografía. Métodos de investigación, Paidós, México, $352 \mathrm{pp}$.

Hilhorst, Dorotea, Lucy Hodgson,

Bram Jansen y Rodrigo Mena

2016 Security Guidelines for Field Research in Complex, Remote and Hazardous Places, International Study for Social Studies / Erasmus University, Rotterdam, 51 pp. <http: / hdl.handle. net/1765/93256> [5 de octubre de 2016].

Howell, NANCY

1990 Surviving Fieldwork. A Report of the Advisory Panel on Health and Safety in Fieldwork, American Anthropological Association, Washington, 217 pp.

Ice, Gillian H., Darna L. Dufour

y NANCY J. STEVEnS

2015 Disasters in Field Research. Preparing for and Coping with Unexpected Events, Rowman and Littlefield, Londres, $216 \mathrm{pp}$.

IFRC

2009 Stay Safe. The International Federation's Guide to a Safer Mission, International Federation of Red Cross and Red Crescent Societies, Ginebra, $214 \mathrm{pp}$.

JAMIESON, JANET

2002 "Negotiating Danger in Fieldwork on Crime: A Researcher's Tale", en Geraldine Lee-Treweek y Stephanie Linkogle (eds.), Danger in the Field. Risk and Ethics in Social Research, Routledge, Londres, pp. 61-71.

Kovats-Bernat, Christopher

2002 "Negotiating Dangerous Fields: Pragmatic Strategies for Fieldwork amid Violence and Terror", en American Anthropologist, vol. 104, núm. 1, marzo, pp. 208-222, DOI: 10.1525/aa. 2002.104.1.208.

LANKSHEAR, GLORIA

2002 "Bacteria and Babies: A Personal Reflection on Researcher Risk in a Hospital", en Geraldine Lee-Treweek y Stephanie Linkogle (eds.), Danger in the Field. Risk and Ethics in Social Research, Routledge, Londres, pp. 72-90.

LEe, Raymond M.

1995 Dangerous Fieldwork, Sage (Qualitative Research Methods Series, 34), Thousand Oaks, 86 pp.

Lee-Treweek, Geraldine

Y Stephanie Linkogle

2002 "Putting Danger in the Frame", en Geraldine LeeTreweek y Stephanie Linkogle (eds.), Danger in the Field. Risk and Ethics in Social Research, Routledge, Londres, pp. 8-25.

Martin-Ortega, Olga y Johanna Herman

2009 "There and Back. Surviving Field Research in Violent and Difficult Situations", en Chandra Lekha Sriram, John C. King, Julie A. Mertus, Olga Martin-Ortega y Johanna Herman (eds.), Surviving Field Research. Working in Violent and Difficult Situations, Routledge, Nueva York, pp. 227-241.

Mertus, Julie A.

2009 "Maintainance if Personal Security, Ethical and Operational Issues", en Chandra Lekha 
Sriram, John C. King, Julie A. Mertus, Olga Martin-Ortega y Johanna Herman (eds.), Surviving Field Research. Working in Violent and Difficult Situations, Routledge, Nueva York, pp. 165- 176 .

Nordstrom, Carolyn

y Antonius C. G. M. RobBen

1995 "The Anthropology and Ethnography of Violence and Sociopolitical Conflict", en Carolyn Nordstrom y Antonius C. G. M. Robben (eds.), Fieldwork under Fire. Contemporary Studies of Violence and Survival, University of California Press, Berkeley, pp. 1-23.

NORMAN, ThOMAS L.

2016 Risk Analysis and Security Countermeasure Selection, CRC Press / Taylor and Francis Group, Nueva York, 449 pp.

SlukA, JefFrey A.

1990 "Participant Observation in Violent Social Contexts", en Human Organization, vol. 49, núm. 2, junio, pp. 114-126, DOI: 10.17730/ humo.49.2.h033174683462676.
SlukA, JEFFrey A.

1995 "Reflections on Managing Danger in Fieldwork", en Carolyn Nordstrom y Antonius C. G. M. Robben (eds.), Fieldwork under Fire. Contemporary Studies of Violence and Survival, University of California Press, Berkeley, pp. 276-294.

TAYLOR, Steven J.

Y ROBERT BOGDAN

1996 Introducción a los métodos cualitativos de investigación. La búsqueda de significados, Paidós, United NATIONS México, 344 pp.

2013 Field Manual, United Nations Disaster Assessment and Coordination, Office for the Coordination of Humanitarian Affairs-United Nations, Nueva York, 269 pp.

WESTMARLAND, LOUISE

2002 "Taking the Flak: Operational Policing, Fear and Violence", en Geraldine Lee-Treweek y Stephanie Linkogle (eds.), Danger in the Field. Risk and Ethics in Social Research, Routledge, Londres, pp. 26-42. 